\title{
Mysteries in Packing Regular Tetrahedra
}

\section{Jeffrey C. Lagarias and Chuanming Zong}

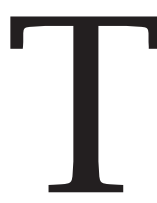

he regular tetrahedron is the simplest Platonic solid. Nevertheless, in studying its packing properties, several renowned scholars have made mistakes, and many questions about it remain unsolved. Currently no one knows the density of its densest packings, the density of its densest translative packings, or the exact value of its congruent kissing number. In this paper we recount historical developments on packing regular tetrahedra, report new results on its translative packing density and congruent kissing number, and formulate several unsolved problems.

\section{Aristotle's Error}

In the history of mathematics, one of the earliest recorded mistakes was made by Aristotle. More than 2,300 years ago, Aristotle (384-322 BCE) taught that the regular tetrahedra fill space. In De Caelo (On the Heavens) Book III.8 he says (in translation): "Among surfaces it is agreed that there are three figures which fill the place that contain them-the triangle, the square and the hexagon: among solids only two, the pyramid and the cube" [3, 306b, p. 319]. Here "pyramid" refers to the regular tetrahedron, a Platonic solid. Thus Aristotle's assertion can be taken to mean: space can be tiled by congruent regular tetrahedra.

Jeffrey C. Lagarias is professor of mathematics at the University of Michigan. His email address is 1agarias@umich. edu.

Chuanming Zong is professor of mathematics at Peking University. His email address is cmzong@math.pku. edu.cn.

DOI: http://dx.doi.org/10.1090/noti918

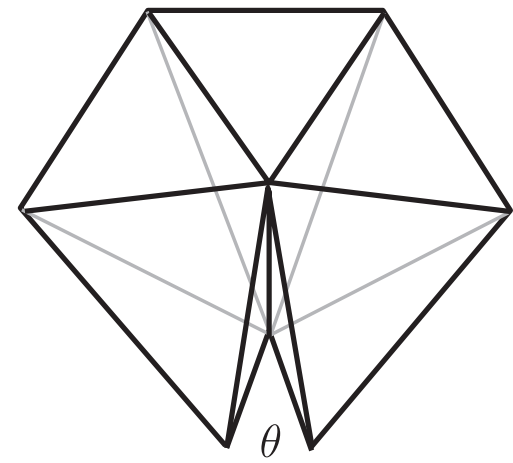

Figure 1. Five regular tetrahedra that share an

edge.

Such a tiling is impossible. Let $T$ denote a regular tetrahedron with unit edges and let $\alpha$ denote one of its dihedral angles. By a routine computation one can deduce that

$$
\alpha=\arccos (1 / 3) \approx 70^{\circ} 32^{\prime} .
$$

If five tetrahedra are fitted around an edge (see Figure 1), then there is a small gap whose angular measure $\theta$ satisfies

$$
0<\theta=2 \pi-5 \alpha \approx 7^{\circ} 21^{\prime}<\alpha,
$$

and we conclude that regular tetrahedra cannot fill the space when arranged face-to-face. In any other tiling arrangement, along an edge a dihedral angle of $\pi-\alpha$ is created, which also cannot be filled by regular tetrahedra.

Of course, at the time of Aristotle, methods of geometric measurement and computation were more limited and computers were not available! 


\section{Eighteen Hundred Years to a Corrigendum}

It took 1,800 years for Aristotle's error to be resolved. Much of the following history was uncovered by Struik [51].

\section{Simplicius and Averroës}

Aristotle's early commentators considered regular tetrahedral packing around a point and in so doing introduced a new mistake.

In the sixth century, to clarify Aristotle's assertion, the commentator Simplicius stated that twelve regular tetrahedra (locally) fill space about a point [33, p. 650, lines 27-28], [51]. Such an arrangement of regular tetrahedra, if it existed, would have twelve tetrahedra touch at a point and fill the solid angle. (But twelve tetrahedra do not fill the solid angle.)

In the twelfth century Averroës (Abu al-Walid Mohammad ibn Ahmad al Rushd (1126-1198)), in Cordoba, wrote thousands of pages of commentary on Aristotle, basing his work on Arabic translations of the Greek. Concerning Aristotle's assertion in De Caelo, he formulated a (mistaken) analysis of trihedral angles as sums of three dihedral angles and, based on this, inferred that twelve tetrahedra fill the same solid angle as eight cubes (they don't). He then concluded that twelve regular tetrahedra (locally) fill space around a point [4, Comm. 66, pp. 628-631].

\section{Michael Scot}

The Arabic commentaries of Averroës reached Christian Europe through Latin translations, many done by the Toledo school of translators. Gerard of Cremona (c. 1114-1187) translated many books, including Ptolemy's Almagest and Euclid's Elements. The translation of the commentaries of Averroës on De Caelo is attributed to Michael Scotus (Michael Scot (1175-c. 1234)) [19, p. 27], [54, pp. 23-25]. In 1217, in Toledo, Scot translated from Arabic the astronomical treatise of Alpetragius (al-Bitrûjî (died c. 1204)), a non-Ptolemaic astronomical system for describing planetary motion [7]. He also translated works of Aristotle and Avicenna on animals. He later joined the service of the Holy Roman Emperor Frederick II as astrologer and wrote several works covering topics on astrology, meteorology, and other subjects [32]. His request to Leonardo of Pisa (Fibonacci) for a copy of Liber Abaci led Fibonacci to prepare his 1228 revised edition, which he dedicated to Scot [50, pp. 15-16]. Scot's knowledge of alchemy and other esoteric subjects (e.g., using the astrolabe to invoke evil spirits but recommending against it) led to his acquiring a reputation as a magician [54, Chap 12]. He appears in Dante's Inferno, condemned to the second lowest circle of Hell [1, Canto XX, v. 115-116]. He is also mentioned in Boccaccio's Decameron, as a necromancer [8, Eighth Day, Story 9].

\section{Scholastic Philosophers: Roger Bacon, Peter of Auvergne, and Thomas Bradwardine}

In 1266-1267 Roger Bacon (c. 1214-1294) wrote a series of essays on natural science at the request of Pope Clement II. According to Struik [51], Bacon writes in "Opus Tertium" [5, Chap. XL, pp. 135-140] that there is a fool in Paris who says that Averroës was incorrect and that twenty pyramids fill space (around a point). Bacon defends the assertion of Averroës that twelve pyramids fill space around a point but concludes that one cannot have complete certainty on these things without constructing the bodies as in Euclid's Book 13. ${ }^{1}$

Thomas Aquinas (c. 1225-1274), best known for his lectures on theology, commented on Aristotle using new, more literal, translations of Aristotle made by William of Moerbeke ${ }^{2}$ (1215-c. 1286). In Naples in 1272-1273 he wrote lectures on Aristotle's De Caelo. These remained incomplete at his death, and the lectures of De Caelo, covering Books III and IV, were completed by Peter of Auvergne (d. 1304) [2, p. 222, bottom]. Peter criticizes Averroës's commentary, saying the assertion of Averroës that twelve tetrahedra fill space (at a point) is contrary to both sense perception and reason and that his assertion on solid angles is not intelligible [2, Book III, Lectio XII, pp. 234-238]. Concerning sense perception, Peter says he saw it for himself. ${ }^{3}$

A perceptive observation was made by Bradwardinus (Thomas Bradwardine (c. 1290-1349)), a scholastic philosopher who late in life became Archbishop of Canterbury. In Geometria Speculativa he stated that there are differing opinions whether the assertion of Averroës that twelve tetrahedra fill space is correct, that others assert that twenty regular tetrahedra (locally) fill space. He observed that a regular icosahedron can be subdivided around its centroid into twenty congruent tetrahedra. But he could not decide if the tetrahedra in this subdivision were regular tetrahedra [44,

\footnotetext{
${ }^{1}$ The culminating Book 13 of The Elements proves existence and uniqueness of the five regular solids and constructs them, in each case inscribed in a sphere.

${ }^{2}$ William of Moerbeke translated part of De Caelo by 1260 , the full text by 1265, and later the Greek commentary of Simplicius, finishing on June 15, 1271 [19, pp. 29 and 48]. He also translated mathematical works of Hero of Alexandria and Archimedes.

3 “Sensui quidem; quoniam si accipiantur duodecim pyramides aequilaterae, et applicentur secundum duodecim angulos circa punctum unum, ad sensum apparebit eas non replere locum corporaliter, et hoc ad sensum expertus sum" [2, p. 237].
} 
Sect. 4.3]. In fact, they are not regular; the solid angle at the central vertex is about ten percent larger than that of a regular tetrahedron.

\section{Regiomontanus and Paul of Middelburg}

Aristotle's error was detected by the fifteenth century. According to Struik [51], the incorrectness of Averroës's assertion, and of Aristotle's, was likely known to Regiomontanus (Johannes Müller von Königsberg (1436-1476)). The work of Regiomontanus covers symbolic algebra, spherical trigonometry, astronomy, and calendar reform. He apparently obtained a correct measure of solid angles, but the results of his investigations were not published. Only a title is known from a catalogue of his posthumous works: "On the five like-sided bodies, that are usually called regular, and which of them fill their natural place, and which do not, in contradiction to the commentator on Aristotle, Averroës." No manuscript copy is known.

In any event, a definite observation of incorrectness was made by Paul of Middelburg (Paulus van Middelburg (1445-1534)), professor of astrology in Padua circa 1478-1481, in astrological prognostics made for the years 1480 and $1481 .^{4}$ He asserts the impossibility of a (local) filling of space using any number of regular tetrahedra, since any such filling would produce a regular polyhedron having the same number of triangular faces, but the only possibilities allowed by Euclid's Book 13, the octahedron and icosahedron, cannot work.

\section{Francesco Maurolico}

A modification that corrects Aristotle's assertion by changing the tiling was found by Francesco Maurolico (1494-1575). He was of Greek descent, became a Benedictine monk in 1521 and later abbot in Messina, Sicily, and eventually was in charge of the Messina mint. He was a mathematician and astronomer and wrote many books, including observations of the supernova in 1572. His Arithmeticorum libri duo [42], published posthumously, contains an early proof by mathematical induction (see Libri [40, pp. 102-118]). His writings include a manuscript with a title nearly identical to the one of Regiomontanus [41]. This manuscript is now lost, but it was catalogued in Rome in 1883 , listing a table of contents and a colophon stating that it was completed on December 9, 1529. According to Libri [40, pp. 242-243], a catalogue of Maurolico's

\footnotetext{
${ }^{4}$ The 1481prognostic reads:

"Correlarium de errore aristotelis et commentatoris eius averroys patum ex 15 propositione 13 euclidis: quia pyramides ex quibus Icocedron conficit non sunt regularis: ergo pyramides regulares non possunt replere locum nedum si 20 sumant: immo nec in quocunque alio numero facerent corpus regulare quod est impossible" ([51, p. 136]).
}

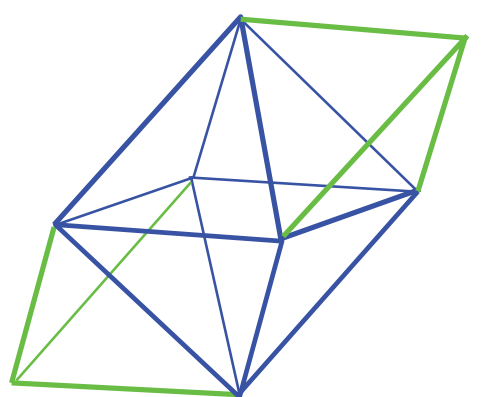

Figure 2. A polyhedral space-filler is obtained from two regular tetrahedra and a regular octahedron.

works lists this one as: "Our booklet on plane and spatial regular figures, which fill their place; although it is certain that Johannes Regiomontanus has written in great detail on this, this work has never been published as far as I know. We show now in this booklet that, of the regular bodies, the cube itself, and pyramids together with octahedra, fill the place whereby it will be clear that Averroës erred in a childish way."

That is, Maurolico knew: there exists a tiling of space using regular polyhedra other than the cube: this tiling is a periodic face-to-face tiling using a mixture of regular tetrahedra and regular octahedra having the same side length (cf. [51]).

To describe it, make a polyhedron by gluing two regular tetrahedra to diagonally opposite faces of a regular octahedron; it has six congruent faces, each a $60^{\circ}-120^{\circ}$ rhombus (see Figure 2). This polyhedron then lattice tiles space using the face-centered cubic (fcc) lattice. The tiling has eight tetrahedra and six octahedra meeting at each vertex, and this tiling is vertex-transitive.

\section{Hilbert's 18th Problem}

In the extended version of his talk presented at the ICM 1900 in Paris, Hilbert [34] proposed twentythree unsolved mathematical problems. At the end of his eighteenth problem, he wrote, "I point out the following question, related to the preceding one, and important to number theory and perhaps sometimes useful to physics and chemistry: How can one arrange most densely in space an infinite number of equal solids of given form, e.g., spheres with given radii or regular tetrahedra with given edges (or in prescribed position); that is, how can

\footnotetext{
5 “De figuris planis, solidisque regularibus locum implentibus libellus noster; quamquam de hoc negocio Ioannem a Regiomonte accuratissime scripsisse certum sit: verum opus nondum, quod sciam, editum. Demonstramus autem in libello e solidis regularibus cubos per se; pyramides vero cum octahedris compactas duntaxat implere locum, qua in re Averroem pueriliter errasse, manifestum erit."
} 
one so fit them together that the ratio of the filled to the unfilled space may be as great as possible?"

The sphere case has a long and complicated history (see Hales [30], [31] and Lagarias [39]). Kepler's conjecture, made in 1611 [37], asserts that the fcc lattice packing is a densest (general) sphere packing. The fcc packing was determined to be the densest lattice sphere packing by Gauss and Seeber in 1831 by studying the arithmetic of positive definite ternary quadratic forms (see [21], [61, Chap. 2]). Perhaps this was one of the reasons for Hilbert to ask this question.

Let $K$ denote a convex body in the Euclidean space $\mathbb{E}^{3}$, with boundary $\partial(K)$, nonempty interior $\operatorname{int}(K)$, and volume $\operatorname{vol}(K)$. We define three notions of packing density as follows. Let $W=\left[-\frac{1}{2}, \frac{1}{2}\right]^{3}$ denote a unit cube centered at the origin so that $s W=\left[-\frac{s}{2}, \frac{s}{2}\right]^{3}$. A general packing $\mathcal{P}$ consists of a (possibly infinite) collection of nonoverlapping congruent copies of $K$, where nonoverlapping means disjoint interiors. For such $\mathcal{P}$ we define an (upper) density

$$
\delta(\mathcal{P}, K):=\limsup _{s \rightarrow \infty} \frac{\operatorname{vol}(\mathcal{P} \cap s W)}{\operatorname{vol}(s W)} .
$$

Then we define the congruent packing density, the translative packing density, and the lattice packing density of $K$ respectively as

$$
\begin{aligned}
\delta^{c}(K) & :=\sup _{\mathcal{P}}\{\delta(\mathcal{P}, K): \mathcal{P} \text { a general packing }\}, \\
\delta^{t}(K) & :=\sup _{\mathcal{P}}\{\delta(\mathcal{P}, K): \mathcal{P} \text { uses translates of } K\},
\end{aligned}
$$

and

$$
\delta^{l}(K):=\sup _{\mathcal{P}}\{\delta(\mathcal{P}, K): \mathcal{P} \text { is a lattice packing }\} .
$$

One can show that each of these suprema is attained: e.g., there exists a general packing $\mathcal{P}$ having

$$
\delta(\mathcal{P}, K)=\delta^{\mathcal{C}}(K),
$$

and similarly there exist a translative packing and lattice packing attaining their respective suprema. Moreover, such $\mathcal{P}$ can also be chosen to have a limiting density, i.e., having the corresponding lower density equal to their upper density. In fact, for $\delta^{c}(K), \delta^{t}(K)$ and $\delta^{l}(K)$, the unit cube $W$ in the definition of $\delta(\mathcal{P}, K)$ can be replaced by any other fixed convex body. For basic results and problems about these densities we refer to [10], [17], [18], [24], [46], and [61].

It follows from these definitions that

$$
\delta^{l}(K) \leq \delta^{t}(K) \leq \delta^{c}(K) \leq 1
$$

hold for every convex body $K$. Let $\sigma(\mathbf{x})$ denote a nonsingular affine linear transformation from $\mathbb{E}^{3}$ to $\mathbb{E}^{3}$. It is known (easy to verify) that both

$$
\delta^{l}(\sigma(K))=\delta^{l}(K)
$$

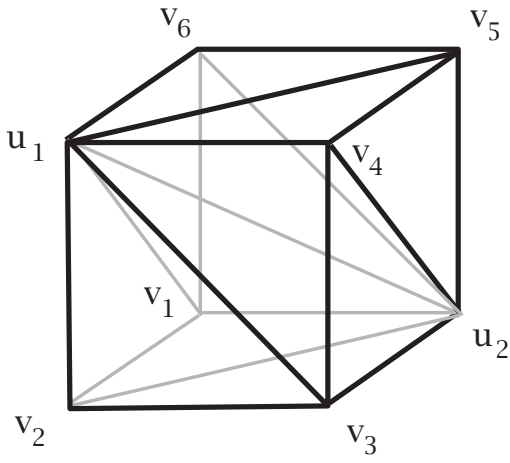

Figure 3. A cube can be divided into six congruent tetrahedra.

and

$$
\delta^{t}(\sigma(K))=\delta^{t}(K)
$$

hold for all $K$ and $\sigma$. However, for some objects $K$ and suitable $\sigma$, we may have

$$
\delta^{c}(\sigma(K)) \neq \delta^{c}(K) .
$$

For example, let $\mathbf{u}_{1}, \mathbf{u}_{2}, \mathbf{v}_{1}, \mathbf{v}_{2}, \ldots, \mathbf{v}_{6}$ be the eight vertices of a unit cube, as shown in Figure 3 , and let $T_{i}$ denote the tetrahedron with vertices $\mathbf{u}_{1}, \mathbf{u}_{2}, \mathbf{v}_{i}$, and $\mathbf{v}_{i+1}$ (we take $\mathbf{v}_{7}=\mathbf{v}_{1}$ in $T_{6}$ ). It is easy to see that the six tetrahedra $T_{1}, T_{2}, \ldots, T_{6}$ are congruent to each other. Thus, since unit cubes tile the space nicely, we have

$$
\delta^{c}\left(T_{1}\right)=1 .
$$

In fact, we obtain a periodic tetrahedron tiling of space by copies of $T_{1}$ having six tetrahedra in its unit cell. On the other hand, for the regular tetrahedron $T$ we have (see [9], p. 208)

$$
\delta^{c}(T)<1,
$$

and therefore

$$
\delta^{c}\left(T_{1}\right) \neq \delta^{c}(T),
$$

although $T_{1}=\sigma(T)$ holds for some suitable affine linear transformation $\sigma$. This illustrates the subtle dependence of the maximal density of a (congruent) tetrahedron packing on the shape of the tetrahedron. It remains an open problem to determine those shapes of tetrahedra whose congruent copies tile space; see Senechal [49].

\section{Minkowski's Genius and Mistake}

Assume that $\mathbf{a}_{1}, \mathbf{a}_{2}, \mathbf{a}_{3}$ are three linearly independent vectors in $\mathbb{E}^{3}$. We call

$$
\Lambda=\left\{\sum z_{i} \mathbf{a}_{i}: z_{i} \in \mathbb{Z}\right\}
$$

a lattice. Lattices are very regular discrete sets in the space, which are additive groups with three generators.

The first work on lattice packing of general convex bodies in $E^{3}$ was done by Minkowski [43] in 1904 . Let $S$ be a centrally symmetric convex body 
centered at the origin o. Minkowski proved the following criterion for densest lattice packings:

Theorem 1. If $S$ has a lattice packing of $S+\Lambda$ of maximal density, then $\Lambda$ has a basis $\mathbf{a}_{1}, \mathbf{a}_{2}$, and $\mathbf{a}_{3}$ such that either

$$
\left\{\mathbf{a}_{1}, \mathbf{a}_{2}, \mathbf{a}_{3}, \mathbf{a}_{1}-\mathbf{a}_{2}, \mathbf{a}_{2}-\mathbf{a}_{3}, \mathbf{a}_{3}-\mathbf{a}_{1}\right\} \subset \partial(2 S)
$$

or

$$
\left\{\mathbf{a}_{1}, \mathbf{a}_{2}, \mathbf{a}_{3}, \mathbf{a}_{1}+\mathbf{a}_{2}, \mathbf{a}_{2}+\mathbf{a}_{3}, \mathbf{a}_{3}+\mathbf{a}_{1}\right\} \subset \partial(2 S) .
$$

As an application, he determined the density of the densest lattice packings of an octahedron $O$. In other words, he proved

$$
\delta^{l}(O)=\frac{18}{19} .
$$

Let $K$ be a convex set and define

$$
D(K)=\{\mathbf{x}-\mathbf{y}: \mathbf{x}, \mathbf{y} \in K\} .
$$

Usually, we call $D(K)$ the difference set of $K$. Clearly $D(K)$ is a centrally symmetric convex set centered at the origin $\mathbf{o}$. It can be shown (see [43]) that

$$
(K+\mathbf{x}) \cap(K+\mathbf{y}) \neq \varnothing
$$

if and only if

$$
\left(\frac{1}{2} D(K)+\mathbf{x}\right) \cap\left(\frac{1}{2} D(K)+\mathbf{y}\right) \neq \varnothing .
$$

Therefore, for a discrete set $X$ in $\mathbb{E}^{3}, K+X$ is a packing if and only if $\frac{1}{2} D(K)+X$ is a packing. Consequently, we get

$$
\delta^{t}(K)=\frac{2^{3} \operatorname{vol}(K)}{\operatorname{vol}(D(K))} \cdot \delta^{t}(D(K))
$$

and

$$
\delta^{l}(K)=\frac{2^{3} \operatorname{vol}(K)}{\operatorname{vol}(D(K))} \cdot \delta^{l}(D(K)) .
$$

In [43, p. 312], Minkowski wrote ${ }^{6}$ "If $K$ is a tetrahedron, then $\frac{1}{2} D(K)$ is an octahedron with faces parallel to the faces of the tetrahedron." Let $O$ denote the regular octahedron of edge length one. By routine computations, one can get

$$
\operatorname{vol}(T)=\sqrt{2} / 12
$$

and

$$
\operatorname{vol}(O)=\sqrt{2} / 3 .
$$

Then, by (2) and (4) Minkowski [43] concluded that

$$
\delta^{l}(T)=\frac{9}{38} .
$$

Minkowski's idea was brilliant. Unfortunately, he made a mistake. The difference set of a regular tetrahedron is not an octahedron but a cuboctahedron. As shown in Figure 4, a cuboctahedron is very different from an octahedron.

6 "Ist z.B. $K$ ein Tetraeder, so wird $\frac{1}{2} D(K)$ ein Oktaeder mit Flächen parallel den Flächen des Tetraeders."

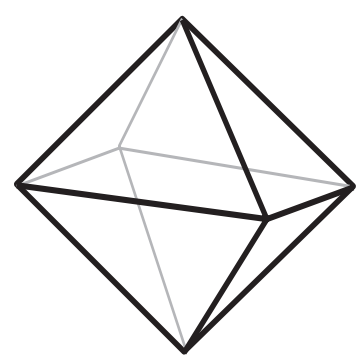

Octahedron

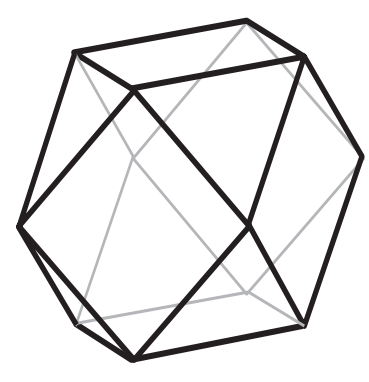

Cuboctahedron
Figure 4. A cuboctahedron is very different from an octahedron.

\section{Groemer's and Hoylman's Work}

Minkowski's mistake was discovered by Groemer [23] in 1962.

Let $T^{\prime}$ denote the tetrahedron with vertices $(-1,1,1),(1,-1,1),(1,1,-1)$ and $(-1,-1,-1)$, and let $\Lambda$ be the lattice generated by $\mathbf{a}_{1}=$ $\left(2,-\frac{1}{3},-\frac{1}{3}\right), \mathbf{a}_{2}=\left(-\frac{1}{3}, 2,-\frac{1}{3}\right)$ and $\mathbf{a}_{3}=\left(-\frac{1}{3},-\frac{1}{3}, 2\right)$. It can be shown that

$$
D\left(T^{\prime}\right)=\left\{\left(x_{1}, x_{2}, x_{3}\right): \sum\left|x_{i}\right| \leq 4,\left|x_{i}\right| \leq 2\right\}
$$

and

$$
\Lambda \cap \operatorname{int}\left(D\left(T^{\prime}\right)\right)=\mathbf{o} .
$$

Therefore both $\frac{1}{2} D\left(T^{\prime}\right)+\Lambda$ and $T^{\prime}+\Lambda$ are lattice packings. ${ }^{7}$ By routine computation it follows that the packing density of $\frac{1}{2} D\left(T^{\prime}\right)+\Lambda$ and $T^{\prime}+\Lambda$ are $\frac{45}{49}$ and $\frac{18}{49}$, respectively. Thus, Groemer [23] obtained

$$
\delta^{l}(D(T)) \geq \frac{45}{49}
$$

and

$$
\delta^{l}(T) \geq \frac{18}{49} .
$$

Clearly Minkowski's conclusion (5) is wrong.

In addition, Groemer also observed that each tetrahedron in $T^{\prime}+\Lambda$ touches fourteen others. However, he was not able to prove that the equalities in (6) and (7) hold.

In 1970 Hoylman [35] applied Minkowski's criterion to a cuboctahedron $C$. By considering thirty-eight cases with respect to the possible positions of the three vectors of the bases, he proved

$$
\begin{aligned}
& \delta^{l}(C)=\frac{45}{49}, \\
& \delta^{l}(T)=\frac{18}{49},
\end{aligned}
$$

and the optimal lattice is unique up to certain equivalence. In fact, Groemer's example is the only optimal lattice tetrahedron packing.

\begin{tabular}{lrlr}
\hline${ }^{7}$ Minkowski's & paper & also & provided \\
$a \quad$ criterion & to & check & whether \\
$K+\Lambda$ is a packing. & & &
\end{tabular}




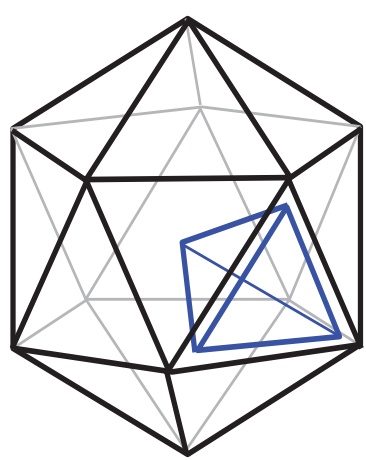

Figure 5. An icosahedron; twenty regular tetrahedra fit in it.

Based on Minkowski's work, in 2000 Betke and Henk [6] developed an algorithm by which one can determine the density of the densest lattice packing of an arbitrary three-dimensional polytope. As an example of application, Hoylman's result was verified.

\section{Tetrahedra Touching a Point}

How many nonoverlapping regular tetrahedra can touch a point?

By considering the solid angle that a regular tetrahedron cuts out, which is

$$
3 \arccos (1 / 3)-\pi \approx 0.55129 \text { steradians, }
$$

and comparing with the full solid angle $4 \pi$, it follows that at most twenty-two tetrahedra can occupy the solid angle without overlap.

One finds that twenty regular tetrahedra can touch without overlap. This fact can be deduced from the structure of an icosahedron, as shown in Figure 5, since the distance between its centroid and vertices is shorter than its edge length. One places the twenty tetrahedra so each has one vertex at its centroid of edge length so that their opposite face sits (concentrically) inside a corresponding face of the icosahedron. There are now small gaps between each of the twenty tetrahedra so arranged.

Conjecture 1. The maximal number of nonoverlapping regular tetrahedra that can meet at a point is twenty.

This problem has circulated in the mathematical community for some years and has been noted by more than one person. Paul Sally (University of Chicago) [47] said that he encountered the question at Lincoln Laboratories in 1958 and later circulated it.

Conjecture 1 can be reformulated as a twodimensional packing problem. It is equivalent to the assertion: The maximum number of equilateral spherical triangles with angle $\arccos (1 / 3)$ that can be placed without overlap on the surface of a sphere is twenty.

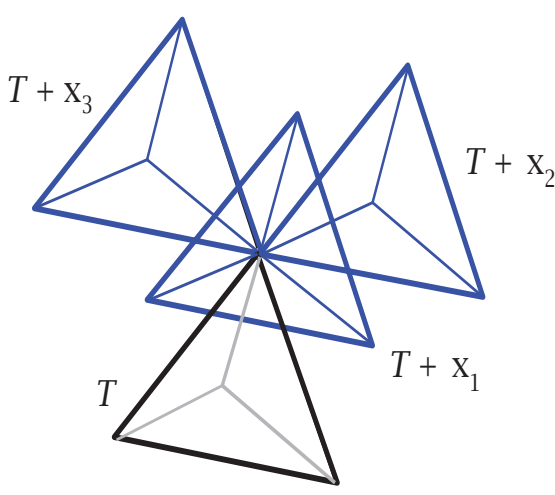

Figure 6. Three tetrahedra meet at a vertex of a fixed tetrahedron.

\section{Kissing Numbers of Tetrahedra}

The kissing number problem concerns finite packings. It was originally raised as the question: How many spheres, all of the same radius, can touch another sphere of the same radius? In the fourteenth century Thomas Bradwardine observed that twelve such spheres can touch a given sphere [44, Sect. 4.42]. The mathematician David Gregory discussed related problems with Isaac Newton in 1694 , and in an unpublished notebook, Gregory recorded the assertion that thirteen spheres can touch one sphere [45, Vol. III, p. 317]. A rigorous mathematical proof that the kissing number of a sphere is twelve was published only in 1953 by Schütte and van der Waerden [48] (see also [9, Chap. 8], [14, Chap. 1], [61, Chap. 1]).

To generalize the thirteen sphere problem of Gregory and Newton, Hadwiger [26] introduced and studied the translative kissing number of a general convex body $K$. Let $\tau^{c}(K), \tau^{t}(K)$ and $\tau^{l}(K)$ denote the congruent kissing number, the translative kissing number, and the lattice kissing number of $K$, respectively. For example, $\tau^{t}(K)$ is the maximal number of nonoverlapping translates of $K$, all of which touch $K$ at its boundary. Clearly, we have

$$
\tau^{l}(K) \leq \tau^{t}(K) \leq \tau^{c}(K) .
$$

In addition, both $\tau^{l}(K)$ and $\tau^{t}(K)$ are invariant under nonsingular linear transformations. Of course, for $\tau^{c}(K)$ this is not always true.

In 1961 Grünbaum [25] made the following conjecture: The translative kissing number of an $n$-dimensional simplex is $n(n+1)$.

This conjecture lasted only one year, as Groemer's example from the last section disproves it. Groemer's example shows that

$$
\tau^{c}(T) \geq \tau^{t}(T) \geq \tau^{l}(T) \geq 14 .
$$

But what are the values of $\tau^{l}(T), \tau^{t}(T)$, and $\tau^{\mathcal{C}}(T)$ ?

For convenience, we assume that one of the four vertices of $T$ is the origin $\mathbf{o}$ and the other three are $\mathbf{v}_{1}, \mathbf{v}_{2}$, and $\mathbf{v}_{3}$. 
When Grünbaum made his conjecture, perhaps he thought about the structure that at each vertex of $T$ there are three translates that touch it (as shown in Figure 6), and therefore altogether twelve translates surround $T$. In fact, this is the local structure of the lattice packing $T+\Lambda_{1}$, where

$$
\Lambda_{1}=\left\{\sum z_{i} \mathbf{v}_{i}: z_{i} \in \mathbb{Z}\right\} .
$$

In 1996 Zong [59] observed that the holes in $T+\Lambda_{1}$ are big enough to hold additional translates of $T$. In fact, we can put a whole copy of $T+\Lambda_{1}$ into it. We write $\mathbf{a}_{1}=\mathbf{v}_{1}, \mathbf{a}_{2}=\mathbf{v}_{2}$, and $\mathbf{a}_{3}=\frac{1}{2}\left(\mathbf{v}_{1}+\mathbf{v}_{3}+\mathbf{v}_{3}\right)$ and define

$$
\Lambda_{2}=\left\{\sum z_{i} \mathbf{a}_{i}: z_{i} \in \mathbb{Z}\right\} .
$$

Then it can be verified that $T+\Lambda_{2}$ is a lattice packing of density $1 / 3$ in which each tetrahedron touches eighteen others. Together with Groemer and Hoylman's result, we have (see [60])

Theorem 2. The density of the densest lattice tetrahedron packings is $18 / 49$, in which each tetrahedron touches fourteen others. There is a lattice tetrahedron packing of density $1 / 3$ in which each tetrahedron touches eighteen others.

Besides this discovery, Zong [59] proved that

$$
\tau^{l}(T)=18
$$

and

$$
18 \leq \tau^{t}(T) \leq 19 .
$$

Three years later, by modifying Zong's method, Talata [53] was able to improve (8) to

$$
\tau^{t}(T)=18 \text {. }
$$

\section{Congruent Kissing Numbers of Tetrahedra}

From the viewpoint of pure mathematics, to determine the value $\tau^{c}(T)$ for the regular tetrahedron is an interesting and challenging problem.

For a lower bound, one can arrange fifty-six congruent regular tetrahedra touching a fixed one $T$ to form an interesting cluster of fifty-seven regular tetrahedra. We follow a construction ${ }^{8}$ given by Chen [12, Chap. 2]. First, put four tetrahedra face-to-face on the original one, forming a cluster $\mathbf{B}_{5}$ of five tetrahedra. Second, around each of the six edges of $T$, insert two more tetrahedra, each face-to-face with one of the four tetrahedra added in the first step. The resulting cluster, $\mathbf{B}_{17}$, has five tetrahedra sharing each edge of $T$, the maximum possible. Third, insert at each vertex new tetrahedra that sit face-to-face with each of the six tetrahedra added at the last step that touch that vertex. This adds twenty-four new nonoverlapping tetrahedra, resulting in a cluster

\footnotetext{
${ }^{8}$ Chen gives explicit constructions with coordinates for all the clusters listed, but does not observe the kissing number property of $\mathbf{B}_{57}$.
}

$\mathbf{B}_{41}$. This cluster is invariant under the symmetric group $S_{4}$ of twenty-four isometries preserving $T$. Finally, there is still room at each vertex to insert four more tetrahedra touching it without overlap; these tetrahedra have some room to move and need not sit face-to-face. This adds sixteen more tetrahedra, giving the desired cluster $\mathbf{B}_{57}$. One may check that this cluster has twenty tetrahedra touching each of the vertices of $T$ and furthermore that the final layer of sixteen tetrahedra may be chosen so that $\mathbf{B}_{57}$ has the full $S_{4}$-symmetry group of isometries. This construction establishes that

$$
\tau^{C}(T) \geq 56 \text {. }
$$

To obtain an upper bound, let $B$ denote the unit ball centered at the origin. If $T^{*}$ is congruent to $T$ and $T \cap T^{*} \neq \varnothing$, then we have

$$
T^{*} \subset T+B \text {. }
$$

Thus we get

$$
\left(\tau^{\mathcal{C}}(T)+1\right) \cdot \operatorname{vol}(T) \leq \operatorname{vol}(T+B) .
$$

By routine computations we get

$$
\begin{aligned}
& \operatorname{vol}(T)=\frac{\sqrt{2}}{12}, \\
& \operatorname{vol}(T+B)= \frac{\sqrt{2}}{12}+\sqrt{3} \\
&+3\left(\pi-2 \arctan \frac{\sqrt{2}}{2}\right)+\frac{4 \pi}{3},
\end{aligned}
$$

and therefore

$$
\tau^{C}(T) \leq\left\lfloor\frac{\operatorname{vol}(T+B)}{\operatorname{vol}(T)}\right\rfloor-1=98 .
$$

In view of the conjecture that at most twenty regular tetrahedra can touch a point, it seems reasonable to guess that the construction above is optimal.

Conjecture 2. The congruent kissing number of the regular tetrahedron is

$$
\tau^{\mathcal{C}}(T)=56 .
$$

The congruent kissing number is not invariant under linear transformations, so it can be considered for tetrahedra of any shape. As the shape of $T$ varies, $\tau^{\mathcal{C}}(T)$ may become arbitrarily large. Consider a tetrahedron $T_{\alpha}$ having one face an equilateral triangle of side length 1 in the $(x, y)$ plane, with centroid located at the origin, and with a fourth vertex along the positive $z$-axis at $(0,0, \alpha)$. As $\alpha$ decreases to 0 , the tetrahedron flattens out. Now rotate the tetrahedron $T_{\alpha}$ so that its equilateral triangular face is parallel to the $(y, z)$ plane. For small $\alpha$ many such rotated tetrahedra can be moved by translations to be stacked in parallel touching the original tetrahedron from below without overlap. Thus the kissing number for $T_{\alpha}$ grows without bound as $\alpha$ approaches 0 .

This leads to the following natural problem. 
Problem 1. Determine the minimal congruent kissing number $\delta^{c}(T)$ among all possible tetrahedral shapes $T$.

In particular one may ask: Does the regular tetrahedron have the minimal congruent kissing number among all shapes of tetrahedra?

\section{A Race for Densest Congruent Packing}

In 2006 Conway and Torquato [15] made a breakthrough in constructing dense congruent tetrahedron packings. Their idea is simple but very efficient.

1. Pack twenty regular tetrahedra into an icosahedron (see Figure 5). The fraction of the icosahedral volume occupied by the tetrahedra can be $0.8567627 \ldots$.

2. Construct a lattice icosahedra packing with maximum density. According to Betke and Henk [6] it is $0.8363574 \ldots$.

Thus we obtain a congruent tetrahedron packing of density approximately

$$
0.8363574 \times 0.8567627 \approx 0.716559 .
$$

In other words, we have

$$
\delta^{c}(T) \geq 0.716559 \ldots
$$

They further observed that, by deforming this packing, one can slightly increase the density, and in this way they found a packing with density approximately 0.717455 .

It was conjectured by Ulam (see p. 135 of [20]) that the maximal density $(0.74048 \ldots)$ for packing congruent spheres is smaller than that for any other convex body. Of course, it makes sense to consider a regular tetrahedron as a possible candidate for a counterexample, as Conway and Torquato [15] did.

However, in 2008, by constructing a cluster of eighteen congruent regular tetrahedra and a suitable lattice packing of the cluster, the lower bound (9) of Conway and Torquato was improved by Chen [11] to

$$
\delta^{c}(T) \geq 0.778615 \ldots
$$

This shows that the regular tetrahedron does not supply a counterexample to Ulam's conjecture.

Packings of tetrahedra have recently become relevant in materials science, in part because it is now possible to manufacture nanomaterials made of small tetrahedra, and these materials may have interesting physical properties. It has become a very active research topic, with materials scientists, physicists, and mathematicians all studying tetrahedral packings.

As one example, the Glotzer lab in chemical engineering at the University of Michigan studied such packings viewed as a fluid of hard tetrahedra, and Haji-Akbari, Engel, and Glotzer [27], [28]

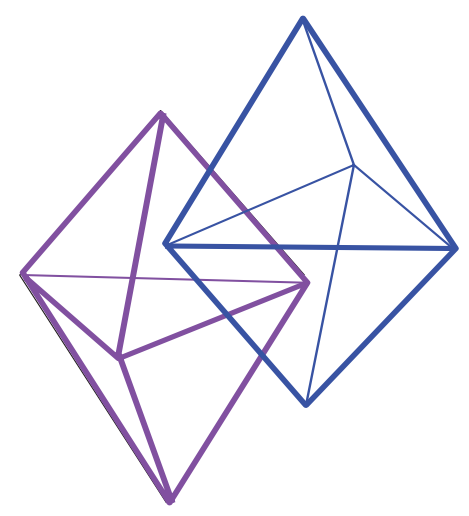

Figure 7. A pair of tetrahedra dimers, each a reflected copy of the other.

computed by simulation its thermodynamic phase diagram. It was observed that such a fluid appears to have a quasicrystalline solid phase at a wide range of pressures and densities [29], [28]. This is the first example of a quasicrystalline phase associated with identical nonspherical particles. Furthermore, this result appears robust against small changes in the shape of the tetrahedra.

Chen's 2008 lower bound was rapidly improved by [55], [56], [29], [36], [57], [13], and [58], some packings being found by computer simulation and others by new constructions.

The current record packing is that of Chen, Engel, and Glotzer [13, Theorem 1]. It is a periodic packing of regular tetrahedra having four tetrahedra in the unit cell, consisting of a dimer of two tetrahedra sharing a face and a reflected copy of this dimer (see Figure 7), and it achieves a packing density $4000 / 4671 \approx 0.856347$. This packing has a set of isometries that act transitively on the individual dimers. It was found as a deformation of a packing of Kallus et al. [36], which itself used a three-dimensional extension of a double-lattice packing idea of Kuperberg and Kuperberg [38]. The paper [13] presents heuristic evidence based on simulation, suggesting that this packing could be the densest packing of regular tetrahedra. In any case, it establishes

$$
\delta^{c}(T) \geq \frac{4000}{4671} \approx 0.856347 \ldots .
$$

While the race for better lower bounds may continue, the first nontrivial upper bound for $\delta^{c}(T)$ was achieved only in 2011. It was proved by Gravel, Elser, and Kallus [22] that

$$
\delta^{c}(T) \leq 1-2.6 \times 10^{-25} .
$$

Congruent tetrahedron packings are still far from being understood. We end this section with two basic problems.

Problem 2. What is the value of $\delta^{c}(T)$ ? Can the optimum be achieved by a periodic packing? 
There is a chance that the packing of Chen, Engel, and Glotzer [13] may be extremal. If true, such a result will likely be very hard to prove. It seems harder than the sphere packing problem, because a proof must take into account the orientation of the tetrahedra in a packing.

Problem 3. Let $\mathcal{F}$ denote the family of all tetrahedra shapes. Is it true that among these the regular tetrahedra are the hardest to pack? That is, do we have

$$
\min _{K \in \mathcal{F}}\left\{\delta^{c}(K)\right\}=\delta^{\mathcal{C}}(T) ?
$$

\section{The Translative Packing Density}

Perhaps it is easier to determine the value of $\delta^{t}(T)$ than the value of $\delta^{c}(T)$. Moreover, since it is invariant under nonsingular affine linear transformations, $\delta^{t}(T)$ is important.

It was proved by Estermann [16] and Süss [52] in 1928 that

$$
\frac{\operatorname{vol}(D(T))}{\operatorname{vol}(T)}=\frac{\operatorname{vol}(C)}{\operatorname{vol}(T)}=20 .
$$

Together with (1), (3), and (7), we can deduce

$$
\frac{18}{49} \leq \delta^{t}(T) \leq \frac{2}{5},
$$

where the lower bound is $0.3673469 \cdots$. Recently, by introducing a particular local method, Zong [62] has proved

$$
\delta^{t}(C) \leq \frac{90 \sqrt{10}}{95 \sqrt{10}-4}=0.9601527 \cdots
$$

and

$$
\delta^{t}(T) \leq \frac{36 \sqrt{10}}{95 \sqrt{10}-4}=0.384061 \cdots .
$$

These facts support the following conjecture.

Conjecture 3. The translative packing density of the regular tetrahedron is

$$
\delta^{t}(T)=\frac{18}{49} .
$$

\section{Acknowledgments}

The work of the first author was supported by NSF Grant DMS-1101373. The work of the second author was supported by 973 Program 2013CB834201, the National Natural Science Foundation of China (No. 11071003), and the Chang Jiang Scholars Program of China. The authors thank J. Bell, E. R. Chen, S. J. Miller, and the reviewers for helpful comments. They also thank M. Senechal for providing an English translation of Struik's paper, and B. Lasall for a translation of Peter of Auvergne's commentary.

\section{References}

1. DANTE AlighieRI, The Inferno, translated by Robert and Jean Hollander, Introduction and Notes by Robert Hollander, Doubleday, New York, 2000.

2. Thomas Aquinas, Opera Omnia, Volumen VigesimumTertium. In Aristotelis Stagiritae Libros Nonnullos Commentaria, vol. 23, Paris, 1875.

3. ARIstotle, On the Heavens, with an English translation by W. K. C. Guthrie, Loeb Classical Library, No. 338, Harvard Univ. Press, Cambridge, MA, 1939.

4. AVERRö̈s, Averrois Cordubensis commentum magnum super libro De celo et mundo Aristotelis, ex recognitiones Frances James Carmody, in lucem edidit Rüdiger Arnzen, Tomus II: Libri II-IV, Indices, Peeters, Leuven, Belgium, 2003.

5. R. BACON, Fr. Rogeri Bacon Opera Quaedam Hactenus Inedita. Vol I. Containing Opus tertium, Opus minus, Compendium philosophiae (J. Brewer, ed.), Longman, Green, Longman and Roberts (series: Rerum Britannicarum medii aevi scriptores, No. 15), Her Majesty's Stationery Office, London, 1859.

6. U. BETKE and M. HENK, Densest lattice packings of 3-polytopes. Comput. Geom. 16 (2000), 157-186.

7. AL-BIṬR ÛĴ̂, De Motibus Celorum: Critical Edition of the Latin Translation of Michael Scot, edited by Francis J. Carmody, Univ. of Calif. Press, Berkeley, CA, 1952.

8. G. BoccAccio, The Decameron, translated by Guido Waldman, Introduction and Notes by Jonathan Usher, Oxford Univ. Press, Oxford, 1993.

9. K. BÖRÖczKY, Finite Packing and Covering, Cambridge Univ. Press, Cambridge, 2004.

10. P. BRAsS, W. MOSER, and J. PACH, Research Problems in Discrete Geometry, Springer-Verlag, New York, 2005.

11. E. R. CHEN, A dense packing of regular tetrahedra, Discrete Comput. Geom. 40 (2008), 214-240.

12. __ A picturebook of tetrahedral packings, Ph.D. Thesis, Univ. of Michigan, 2010.

13. E. R. Chen, M. Engel, and S. C. Glotzer, Dense crystalline dimer packings of regular tetrahedra, Discrete Comput. Geom. 44 (2010), 253-280.

14. J. H. Conway and N. J. A. SloAne, Sphere Packings, Lattices and Groups, Springer-Verlag, New York, 1988; 3rd ed., 1999.

15. J. H. ConwAY and S. Torquato, Packing, tiling, and covering with tetrahedra, Proc. Natl. Acad. Sci. USA 103 (2006), 10612-10617.

16. T. EstermanN, Über den Vektorenbereich eines konvexen Körper, Math. Z. 28 (1928), 471-475.

17. G. FeJEs Tóth and W. KuPERBERG, Packing and covering with convex sets, Handbook of Convex Geometry (edited by P. M. Gruber and J. M. Wills), North-Holland, Amsterdam, 1993, pp. 799-860.

18. L. FEJES TóTH, Lagerungen in der Ebene, auf der Kugeln und im Raum, Springer-Verlag, Berlin, 1953; 2nd ed., 1972.

19. G. GALlE, Peter of Auvergne, Questions on Aristotle's "De Caelo". A Critical Edition with an Interpretative Essay, Leuven Univ. Press, Leuven, Belgium, 2003.

20. M. GARDNER, The Colossal Book of Mathematics, Norton, New York, 2001.

21. C. F. GAUSS, Untersuchungen über die Eigenschaften der positiven ternären quadratischen Formen von Ludwig August Seeber, Göttingische gelehrte Anzeigen (July 9, 1831); J. Reine Angew. Math. 20 (1840), 312-320; Werke, II (1876), 188-196. 
22. S. GRAVEL, V. ELSER, and Y. KALLUS, Upper bound on the packing density of regular tetrahedra and octahedra, Discrete Comput. Geom. 46 (2011), 799-818.

23. H. GROEMER, Über die dichteste gitterförmige Lagerung kongruenter Tetraeder, Monatsh. Math. 66 (1962), 1215.

24. P. M. GRUBER, Convex and Discrete Geometry, SpringerVerlag, Berlin, 2007.

25. B. GRÜNBAUM, On a conjecture of H. Hadwiger, Pacific J. Math. 11 (1961), 215-219.

26. H. HADWIGER, Über Treffanzahlen bei translationsgleichen Eikörpern, Arch. Math. 8 (1957), 212-213.

27. A. HAJI-AKBARI, Thermodynamics of the hard tetrahedron system, Ph.D. Thesis, Univ. of Michigan, 2011.

28. A. Haji-Akbari, M. Engel, and S. C. Glotzer, Phase diagram of hard tetrahedra, J. Chemical Physics 135 (2011), 194101.

29. A. Haji-AKbari, M. Engel, A. S. Keys, X. Zheng, R. G. PetscheK, P. PAlfFy-Muhoray, and S. C. Glotzer, Disordered, quasicrystalline and crystalline phases of densely packed tetrahedra, Nature 462 (2009), 773.

30. T. C. HALES, Cannonballs and honeycombs, Notices Amer. Math. Soc. 47 (2000), 440-449.

31. A proof of the Kepler conjecture, Ann. Math. (2) 162 (2005), 1065-1185.

32. C. H. HASKINS, Michael Scot and Frederick II, Isis, vol. 4, 1921/1922, pp. 250-275.

33. I. L. Heiberg, ed., Simplicii in Aristotelis de Caelo Commentaria, in Commentaria in Aristotelem Graeca, Band VII, Preussische Akademie der Wissenschaften, 1894.

34. D. HilberT, Mathematische Probleme, Arch. Math. Phys. 3 (1901), 44-63; Bull. Amer. Math. Soc. (N.S.) 37 (2000), 407-436.

35. D. J. Hoylman, The densest lattice packing of tetrahedra, Bull. Amer. Math. Soc. 76 (1970), 135-137.

36. Y. KAllus, V. ElSER, and S. GRAVEL, Dense periodic packings of tetrahedra with small repeating units, Discrete Comput. Geom. 44 (2010), 245-252.

37. J. KePler, Strena Seu de Niue Sexangula, Tampach: Frankfort on Main, 1611. English translation: J. Kepler, The Six-Cornered Snowflake (Colin Hardie, Translator), Oxford Univ. Press, Oxford, 1966.

38. G. Kuperberg and W. KuperberG, Double-lattice packings of convex bodies in the plane, Discrete Comput. Geom. 5 (1990), 389-397.

39. J. C. LAGARIAS, The Kepler conjecture and its proof, Chap. 1 in The Kepler Conjecture: The Hales-Ferguson Proof, by Thomas C. Hales, Samuel P. Ferguson (J. C. Lagarias, ed.), Springer-Verlag, New York, 2011.

40. G. LIBRI, Histoire des Sciences Mathématiques en Italie, depuis la renaissance des lettres. Tome Troisiéme, Jules Renouard et Cie, Paris, 1840.

41. F. MAUROLICO, De qvinque solidis, qvaue vvlgo regvlaria dicvntvr, quae videlicet eorvm locvm impleant et qvae non, contra commentatore Aristotelis Averroem, Manuscript. Catalogued in Bibloth. S. Pataleo, in Bibl. Naz. "Vittorio Emauele II", Rome 14 July 1883, listing table of contents.

42. D. Francisci Mavrolyci, Abbatis Messanensis, Arithmeticorvm Libri Dvo, nvnc primvm in lvcem editi, cum rerum omnium notabilium. Indice copiosissimo. Venetijs, apud Franciscum Franciscium Senensem, 1575.
43. H. MinKowsKi, Dichteste gitterförmige Lagerung kongruenter Körper, Nachr. K. Ges. Wiss. Göttingen (1904), 311-355.

44. G. Molland, Thomas Bradwardine, Geometrica Speculativa, Latin text and English translation, with an Introduction and a Commentary, Boethius Ser., Band 18, Stuttgart, Steiner Verl., Wiesbaden, 1989.

45. I. NewTon, The Correspondence of Isaac Newton (9 volumes) (H. W. Turnbull, editor), Cambridge Univ. Press, Cambridge, 1961.

46. C. A. Rogers, Packing and Covering, Cambridge Univ. Press, Cambridge, 1964.

47. P. SALLY, private communication.

48. K. SChÜTTE and B. L. VAN DER WAERDEN, Das Problem der dreizehn Kugeln, Math. Ann. 125 (1953), 325-334.

49. M. SENECHAL, Which tetrahedra fill space? Math. Mag. 54 (1981), 227-243.

50. L. E. SigleR, Fibonacci's Liber Abaci. A Translation into Modern English of Leonardo Pisano's Book of Calculation, Springer-Verlag, New York, 2002.

51. D. J. STRUiK, Het Probleem 'De impletione loci' (Dutch), Nieuw Archief voor Wiskunde, Series 2, 15 (1925-1928), no. 3, 121-137.

52. W. Süss, Über den Vektorenbereich eines Eikörpers, Jber. Deutsch. Math. Verein. 37 (1928), 359-362.

53. I. TALATA, The translative kissing number of tetrahedra is 18, Discrete Comput. Geom. 22 (1999), 231-248.

54. L. THORNDIKE, Michael Scot, Thomas Nelson and Sons, London, 1965.

55. S. TorquAto and Y. JiAo, Dense packings of the Platonic and Archimedean solids, Nature 460 (2009), 876.

56. _ Dense packings of polyhedra: Platonic and Archimedean solids, Phys. Rev. E 80 (2009), 041104.

57. Analytical constructions of a family of dense tetrahedron packings and the role of symmetry, arXiv: 0912 . 4210.

58. __ Exact constructions of a family of dense periodic packings of tetrahedra, Phys. Rev. E (3) 81 (2010), 041310.

59. C. ZonG, The kissing numbers of tetrahedra, Discrete Comput. Geom. 15 (1996), 251-264.

60. Strange Phenomena in Convex and Discrete Geometry, Springer-Verlag, New York, 1996.

61. Sphere Packings, Springer-Verlag, New York, 1999.

62. tetrahedra and cuboctahedra, arXiv: 1208.0420. 